\title{
Discrepancy in rating health-related quality of life of depression between patient and general population
}

\author{
Sylvia A. H. Gerhards • Silvia M. A. A. Evers • \\ Philip W. M. Sabel • Marcus J. H. Huibers
}

Accepted: 6 September 2010/Published online: 30 September 2010

(C) The Author(s) 2010. This article is published with open access at Springerlink.com

\begin{abstract}
Objective To compare the quality of life valuation of a hypothetical depression health state between a general population versus a depressive patient population, and within a patient population.

Method In a self-administered Internet questionnaire, both patient and general population groups filled in the Euroqol 5D (EQ-5D) questionnaire for a hypothetical depression health state describing mild $(N=740)$, moderate $(N=691)$, or severe $(N=670)$ depression and valued it using a rating scale (RS). The 'patient' group consisted of people reporting depressive complaints on the Internet questionnaire, subdivided into three depression severity groups (mild, moderate, severe) based on the Beck Depression Inventory Second Edition (BDI-II) and two groups according to self-perceived duration ( $\leq 3$ months, $\geq 4$ months) of depressive complaints (SPDD).

Results Significant differences were found between the patient and general population and within the patient population on RS outcomes and on the Euroqol domains usual activities, pain/discomfort, and anxiety/depression. The direction of the differences shows that subgroups with
\end{abstract}

S. A. H. Gerhards $(\bowtie) \cdot$ M. J. H. Huibers

Department of Clinical Psychological Science, Faculty

of Psychology, Maastricht University, P.O. Box 616,

6200 MD Maastricht, The Netherlands

e-mail: S.Gerhards@maastrichtuniversity.nl

S. A. H. Gerhards - S. M. A. A. Evers

School for Public Health and Primary Care: Caphri,

Maastricht University, Maastricht, The Netherlands

S. M. A. A. Evers - P. W. M. Sabel

Department of Health Organization, Policy and Economics,

Faculty of Health, Medicine and Life Sciences,

Maastricht University, Maastricht, The Netherlands higher severity or longer duration of depressive complaints value depression health states worse compared with subgroups with less severe complaints, a shorter duration, or no depressive complaints. Moreover, the discrepancy in valuation of a health state between different subgroups changes according to the severity of the health state described.

Conclusion There are discrepancies in the valuation of a hypothetical depression health state between a patient and general population, whereby depression leads to a worse valuation. But also within the patient population, the valuation differs according to depression severity and duration. Identification with the hypothetical health state description might explain the varying differences found between subgroups for the different hypothetical health state descriptions.

Keywords Depression - Rating scale $\cdot$ Health-related quality-of-life $\cdot$ EQ-5D · Patient preferences .

Population preferences

\author{
Abbreviations \\ BDI-II Beck depression inventory second edition \\ EQ-5D Euroqol 5D \\ RS Rating scale \\ SPDD Self-perceived duration of depressive complaints \\ VAS Visual analogue scale
}

\section{Background}

Many studies have shown that depression has an important influence on a patient's health-related quality of life [1-6]. Health-related quality of life is often a main outcome 
parameter in economic evaluations of health care interventions (i.e., cost-utility analysis). However, there is no agreement on who should value the health state's quality of life: the general population, the patient, or some other rater group (e.g., health professionals) [7].

We are aware of only one study on depression comparing a patient population with general population's valuation of a hypothetical health state [8]. This study found that patients reported lower preferences-indicating that patients perceived depression worse than the general population - and there was a trend for a decreasing preference as the depression severity of the patient increased [8]. This seems in contradiction to previous research on other disorders: many studies showed a general tendency that general population scored higher preferences for a health state than patients [7], and others concluded that overall there are no significant differences [9]. In this study, we compare the view of a depressive patient versus general population group on a hypothetical depression health state.

\section{Method}

\section{Recruitment of participants}

Data were collected in a screening to recruit participants for a trial study on depression treatment [10-12]. A random selection of inhabitants in the Southern part of the Netherlands (age 18-65) was sent an invitation letter with unique login codes to complete a screening questionnaire via the Internet.

\section{Creation of the health state descriptions}

For this study, three hypothetical depression states were created: (1) mild, (2) moderate, and (3) severe depressive complaints. The hypothetical states were described by means of the DSM-IV criteria [13] and created based on data of the first 8,916 screening respondents: It was assessed whom of them had depressive complaints (i.e., a cutoff score of 4 on the Beck Depression Inventory for Primary Care [14] and having complaints for at least 3 months), and their data regarding the nine depression complaints according to the DSM-IV criteria and severity of depression (complaints and severity assessed by the Diagnostic Inventory for Depression $[15,16]$ ) were used to compile the three different hypothetical depression states. The hypothetical mild depression state was composed of the four most prevalent complaints of screening respondents with mild complaints $(n=140)$. The hypothetical moderate depression state was composed of the six most prevalent complaints of screening respondents with moderate complaints $(n=260)$. The hypothetical severe depression state was composed by all DSM-IV depression complaints [13].

\section{Valuation procedure}

Respondents were only presented one of the health states of Table 1. After a description and explanation of the questionnaire, the respondent was asked to fill in the EuroQol 5D (EQ-5D) for that hypothetical health state. The EQ-5D consists of five health state dimensions (mobility, self-care, usual activity, pain/discomfort, and anxiety/depression) on which the respondent has to indicate the health state choosing from three levels (no problem, some problems, and major problems) [17, 18]. A last question of the EuroQol is a visual analogue scale (VAS) thermometer ranging from 0 (worst) to 100 (most optimal) on which respondents have to indicate how good or bad the health state is. Due to limitations of the computer system, an adjusted version of the VAS was used, being a rating scale (RS) question to give a rating from 0 to 100 .

Table 1 Hypothetical health state descriptions

Health state description of severe depression

A person is having/suffering from severe depressive complaints. Almost daily he is having a depressed mood and almost no interest or pleasure in (almost) all activities. Besides, he is having the following complaints:

Problems with appetite or changes in weight

Problems with sleeping

Restlessness or being inhibited

Fatigue, loss of energy

Feelings of worthlessness or excessive guilt

Diminished ability to think or concentrate, indecisiveness

Recurrent thoughts of death or suicide

Health state description of moderate depression

A person is having/suffering from moderate depressive complaints. Almost daily he is having a depressed mood. Besides, he is having the following complaints:

Problems with sleeping

Restlessness or being inhibited

Fatigue, loss of energy

Feelings of worthlessness or excessive guilt

Diminished ability to think or concentrate, indecisiveness

\section{Health state description of mild depression}

A person is having/suffering from severe depressive complaints. Almost daily he is having a depressed mood. Besides, he is having the following complaints:

Restlessness or being inhibited

Fatigue, loss of energy

Diminished ability to think or concentrate, indecisiveness 


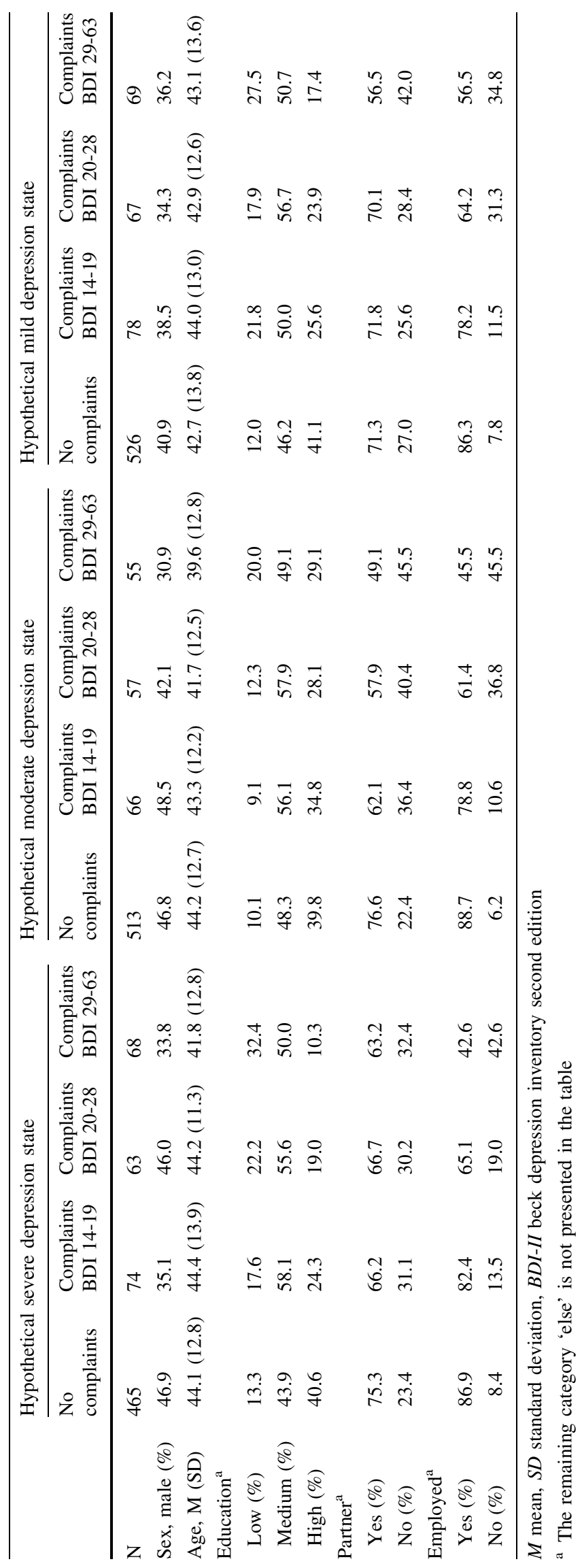




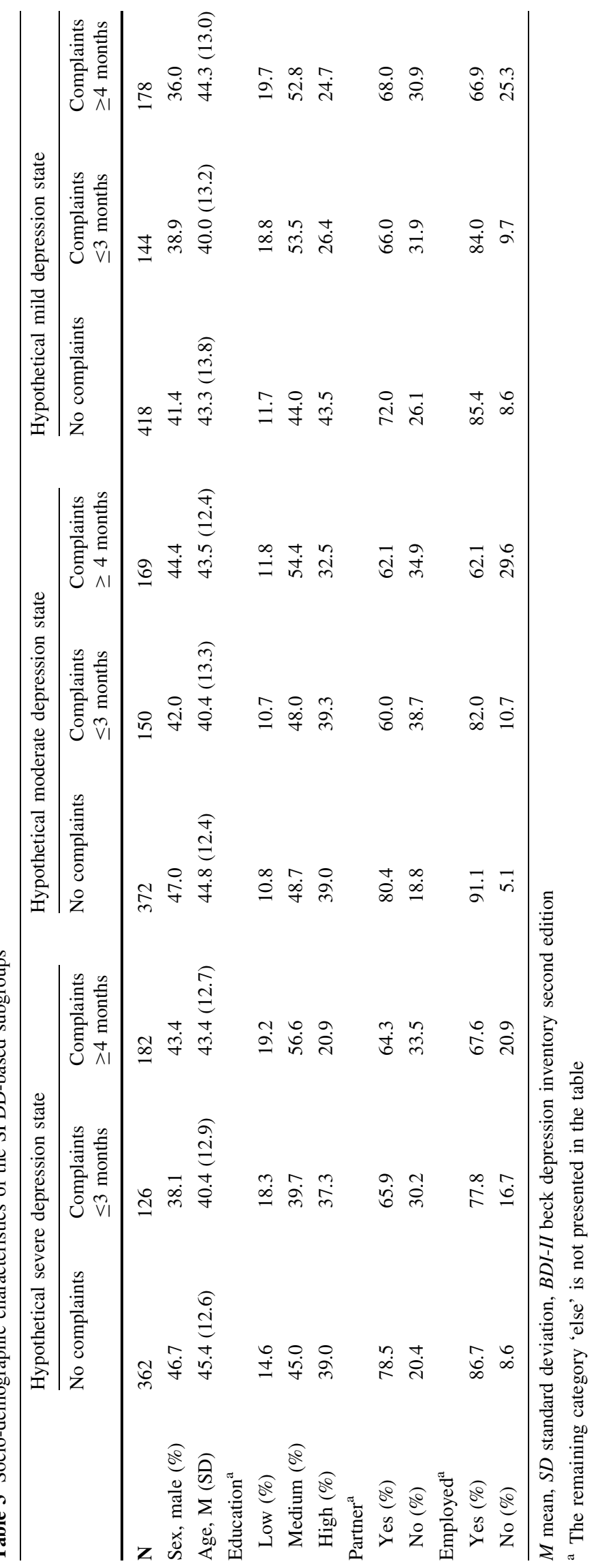


Defining general population and patient subgroups

General population and patient subgroups were created according to two different perspectives: A division was made on depression severity measured by a standardized questionnaire and on the participant's self-perceived duration of complaints.

Depression severity was measured with the Beck Depression Inventory Second Edition (BDI-II) [19-21]. For patients diagnosed with depression, a BDI-II score of 0-13 is categorized 'minimal', 14-19 'mild', 20-28 'moderate severe', and 29-63 'severe' depression [21]. Based on these cutoff scores, we divided four groups: (0) no complaints (i.e., the general population) and (1) mild, (2) moderate, and (3) severe complaints.

The self-perceived duration of depressive complaints (SPDD) was measured by the question: Are you suffering from sadness or depression? According to the answers on this question, three subgroups were divided: (0) no complaints (i.e., the general population), (1) depressive complaints up to about 3 months, and (2) depressive complaints for longer than 3 months.

\section{Analysis}

Analyses are preformed on the BDI-II-based subgroups and on the SPDD-based subgroups. First, the sociodemographic characteristics of the subgroups were evaluated (mean and standard deviation, or percentage scores). Secondly, differences between subgroups on the Euroqol dimensions are assessed by the Chi-square test. Thirdly, the RS scores of the subgroups are evaluated (mean score and standard deviation) and differences between subgroups are tested by the Mann-Whitney test $(P<0.05)$.

\section{Results}

Study participants

In total, 2,101 respondents completed the questionnaire, of which 670 of were presented the severe health state, 691 the moderate, and 740 the mild health state. Tables 2 and 3 present the baseline characteristics of these participants per subgroup. The results indicate that subgroups without depressive complaints have relatively more male, employed, higher educated respondents with partner as opposed to the subgroups with depressive complaints.

\section{Differences in Euroqol domain scores}

The $P$-values of the Chi-square tests show significant differences between the subgroups' scores on the EQ-5D

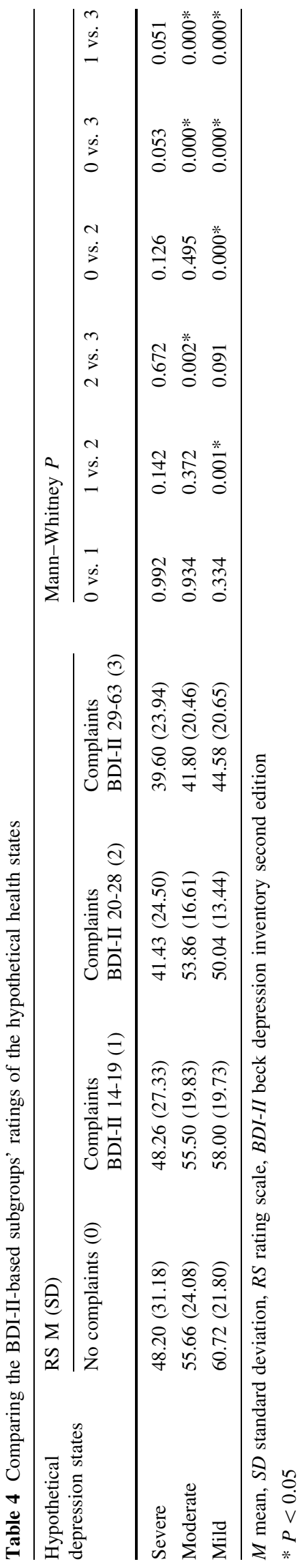


Table 5 Comparing the SPDD-based subgroups' ratings of the hypothetical health states

\begin{tabular}{|c|c|c|c|c|c|c|}
\hline \multirow{2}{*}{$\begin{array}{l}\text { Hypothetical } \\
\text { depression states }\end{array}$} & \multicolumn{3}{|l|}{ RS M (SD) } & \multicolumn{3}{|c|}{ Mann-Whitney $P$} \\
\hline & No complaints $(0)$ & Complaints $\leq 3$ months & Complaints $\geq 4$ months (2) & 0 vs. 1 & 1 vs. 2 & 0 vs. 2 \\
\hline Severe & $48.74(32.02)$ & $44.29(28.17)$ & $44.30(25.21)$ & 0.153 & 0.962 & 0.150 \\
\hline Moderate & $55.75(24.94)$ & $56.80(20.70)$ & $49.26(20.34)$ & 0.714 & $0.002 *$ & $0.003^{*}$ \\
\hline Mild & $60.89(21.74)$ & $57.17(21.61)$ & $51.72(19.37)$ & 0.130 & $0.008 *$ & $0.000 *$ \\
\hline
\end{tabular}

$M$ mean, $S D$ standard deviation, $S P D D$ self-perceived duration of depressive complaints, $R S$ rating scale

* $P<0.05$

domains for all three hypothetical depression health states. The higher the BDI-II score or the longer the duration of complaints according to the SPDD of the subgroups' respondents, the more problems are reported on the domains 'usual activities', 'pain/discomfort', and 'anxiety/ depression'. For the domain 'self-care', there were only significant differences between the SPDD-subgroups, whereby subgroups with a longer duration of complaints report less problems on this domain compared to subgroups without depressive complaints or a shorter duration of complaints. The subgroups do not show significant differences on the domain 'mobility'.

\section{Differences in RS}

Tables 4 and 5 present the mean RS outcomes for each hypothetical health state. Results indicate that a more severe health state is given relatively lower RS scores compared with a less severe hypothetical depression state. The RS outcome for the severe health state description does not differ significantly between the subgroups. Although Table 4 shows a trend toward significance when comparing the severely depressed subgroup with the subgroup with mild complaints $(P=0.051)$ or the subgroup without complaints $(P=0.053)$. Within the BDI-II-based subgroups, the following significant differences were found: For the moderate health state description, the outcomes of the severely depressed subgroup (BDI-II 29-63) differ significantly from all other subgroups (all $P<0.05$ ). For the mild health state description, the RS outcomes from the severely depressed subgroup (BDI-II 29-63) and the moderately depressed subgroup (BDI-II 20-28) differ significantly from the subgroup without complaints and from the subgroup with mild complaints (BDI 14-19).

Within the SPDD-based subgroups, the following significant differences were found: For the moderate health state description, the RS scores of the subgroup with complaints $\geq 4$ months differ significantly from the subgroup with complaints $\leq 3$ months $(P=0.002)$ and from the subgroup without complaints $(P=0.003)$. For the mild health state description, the RS outcomes of the subgroup $\geq 4$ months as well differ significantly from the subgroup with complaints $\leq 3$ months $(P=0.008)$ and from the subgroup without complaints $(P=0.000)$.

\section{Discussion}

People with a higher severity or longer duration of depressive complaints value depression health states worse compared with people with less severe complaints, a shorter duration, or no depressive complaints. Moreover, the discrepancy in valuation of a health state between different subgroups changes according to the severity of the health state described, which might be explained by identification with the health state described: There seems to be a relation between the severity of the respondents' own depression complaints and the severity of the hypothetical health state description to be valued. The point from which on groups differ in the valuation of the description changes with the severity of complaints described. For the mild health state description, a significant difference from the general population RS score is found for respondents with at least moderate complaints; for the moderate health state description, this difference is found for respondents with at least severe complaints; and for the severe health state description, this difference is not found for any subgroups. This might indicate that those with at least moderate complaints recognize themselves in the description of mild depression, and those with severe complaints recognize themselves in the description of moderate depression, while the description of severe depression might be too severe for identification in all subgroups.

The fact that differences were also found between the SPDD-based patient subgroups might be related to the aspects of adaptation, response shift, or shift in internal standards. These aspects are often given as explanations for differences in valuations between a patient and general population [22]: Patients who experience complaints for a longer period of time might have more adaptation or shifted further opposed to those who only recently started to experience the health complaints.

A limitation of this study is that we did not assess the contribution of severity and duration simultaneously. 
We found large Spearman correlations between the SPDD and BDI-II variables subdivided into the subgroup categories presented in this study $(r=0.684,0.600$, and 0.641 for, respectively, the severe, moderate, and mild health state description). However, since each severity category can be combined with each duration category, integrating the severity and duration categories would imply a larger number of subgroups and lead to some very small subgroups.

Acknowledgments We thank Esther de Graaf, Annie Hendriks and Greet Kellens for their assistance in recruitment and data-collection, and Rosanne Janssen for the development of the infrastructure for online data-collection. This study collected data within the screening for a trial financed by ZonMw (Netherlands Organisation for Health Research and Development; project number 945-04-417), research institute EPP and research institute CAPHRI. Municipalities Eijsden, Meerssen, Sittard-Geleen, Valkenburg and Maastricht sponsored the trial study. The study sponsors had no role in the design of the study; in the collection, analysis, and interpretation of the data; in the writing of the report; and in the decision to submit the article for publication.

Conflict of interest All authors declare that they have no conflicts of interest.

Open Access This article is distributed under the terms of the Creative Commons Attribution Noncommercial License which permits any noncommercial use, distribution, and reproduction in any medium, provided the original author(s) and source are credited.

\section{References}

1. ten Doesschate, M. C., Koeter, M. W. J., Bockting, C. L. H., Schene, A. H., \& Group, T. D. S. (2010). Health related quality of life in recurrent depression: A comparison with a general population sample. Journal of Affective Disorders, 120, 126-132.

2. Bijl, R. V., \& Ravelli, A. (2000). Current and residual functional disability associated with psychopathology: Findings from the Netherlands mental health survey and incidence study (NEMESIS). Psychological Medicine, 30, 657-668.

3. Kruijshaar, M. E., Hoeymans, N., Bijl, R. V., Spijker, J., \& Essink-Bot, M. L. (2003). Levels of disability in major depression. Findings from the Netherlands mental health survey and incidence study (NEMESIS). Journal of Affective Disorders, 77(1), 53-64.

4. Creed, F., Morgan, R., Fiddler, M., Marshall, S., Guthrie, E., \& House, A. (2002). Depression and anxiety impair health-related quality of life and are associated with increased costs in general medical inpatients. Psychosomatics, 43(4), 302-309.

5. Rapaport, M. H., Clary, C., Fayyad, R., \& Endicott, J. (2005). Quality-of-life impairment in depressive and anxiety disorders. American Journal of Psychiatry, 162(6), 1171-1178.

6. Sobocki, P., Ekman, M., Agren, H., Krakau, I., Runeson, B., Martensson, B., et al. (2007). Health-related quality of life measured with EQ-5D in patients treated for depression in primary care. Value in Health, 10(2), 153-160.
7. de Wit, G. A., Bussbach, J. J. V., \& De Charro, F. T. (2000). Sensitivity and perspective in the valuation of health status: Whose values count? Health Economics, 9, 109-126.

8. Pyne, J. M., Fortney, J. C., Tripathi, S., Feeny, D., Ubel, P., \& Brazier, J. (2009). How bad is depression? Preference score estimates from depressed patients and the general population. Health Services Research, 44(4), 1406-1423.

9. Dolders, M. G. T., Zeegers, M. P. A., Groot, W., \& Ament, A. (2006). A meta-analysis demonstrates no significant differences between patient and population preferences. Journal of Clinical Epidemiology, 59, 653-664.

10. Gerhards, S. A. H., de Graaf, L. E., Jacobs, L. E., Severens, J. L., Huibers, M. J. H., Arntz, A., et al. (2010). Economic evaluation of online computerised cognitive-behavioural therapy without support for depression in primary care: Randomised trial. British Journal of Psychiatry, 196(4), 310-318.

11. de Graaf, L. E., Gerhards, S. A. H., Evers, S. M. A. A., Arntz, A. R., Riper, H., Severens, J. L., et al. (2008). Clinical and costeffectiveness of computerised cognitive behavioural therapy for depression in primary care: Design of a randomised trial. $B M C$ Public Health, 8, 224.

12. de Graaf, L. E., Gerhards, S. A. H., Arntz, A. R., Riper, H., Metsemakers, J. F. M., Evers, S. M. A. A., et al. (2009). Clinical effectiveness of online computerized cognitive behavioural therapy without support for depression in primary care: A randomized trial. British Journal of Psychiatry, 195(1), 73-80.

13. American Psychiatric Association. (2000). DSM-IV-TR. Diagnostic and statistical manual of mental disorders (4th ed.). Washington, DC: American Psychiatric Press.

14. Steer, R. A., Cavalieri, T. A., Leonard, D. M., \& Beck, A. T. (1999). Use of the beck depression inventory for primary care to screen for major depressive disorders. General Hospital Psychiatry, 21, 106-111.

15. Zimmerman, M., Sheeran, T., \& Young, D. (2004). The diagnostic inventory for depression: A self-report scale to diagnose DSM-IV major depressive disorder. Clinical Psychology, 60(1), 87-110.

16. Sheeran, T., \& Zimmerman, M. (2002). Case identification of depression with self-report questionnaires. Psychiatry Research, 109, 51-59.

17. Brooks, R. (1996). EuroQol: The current state of play. Health Policy, 37(1), 53-72.

18. EuroQol Group. (1990). EuroQol-a new facility for the measurement of health-related quality of life. Health Policy, 16(3), 199-208.

19. Beck, A. T., Steer, R. A., Ball, R., \& Ranieri, W. F. (1996). Comparison of beck depression inventories-IA and -II in psychiatric outpatients. Journal of Personality Assessment, 67(3), 588-597.

20. Arnau, R. C., Meagher, M. W., Norris, M. P., \& Bramson, R. (2001). Psychometric evaluation of the beck depression inventory-II with primary care medical patients. Health Psychology, 20(2), 112-119.

21. Van der Does, A. J. W. (2002). De Nederlandse versie van de beck depression inventory-second edition (BDI-II-NL): Handleiding [The Dutch version of the Beck Depression Inventorysecond edition (BDI-II-NL): Manual]. Enschede: The Psychological Corporation.

22. Ubel, P. A., Loewenstein, G., \& Jepson, C. (2003). Whose quality of life? A commentary exploring discrepancies between health state evaluations of patients and the general public. Quality of Life Research, 12, 599-607. 